Materials science is a gateway to minimizing energy consumption and water consumption, and understanding interfaces is one of the keys that unlocks it. Sensor technology, catalysis, and membrane science are only some of the fields that can contribute to the water-energy challenge.

\title{
Crises and opportunities at the energy-water interface
}

\author{
By Eva Karatairi \\ Feature Editor: Seth B. Darling
}

$\mathrm{F}$ or many, water and energy are considered amenities people can command with the tips of their fingers. But easy access to water and energy is neither universal, nor has it been easily available for much more than half a century for many people in rural and urban areas around the world. Scientists and engineers, with the help of gravity and electricity, have pushed water up and down mountains and across plains to reach home faucets as well as industries and fields, and have treated it to improve its quality, eventually returning it to the environment without posing a major threat to humans or nature. In turn, water has played a big part in energy production by cooling thermal power plants, putting in motion generators in hydroelectric facilities, stimulating oil and gas recovery, and refining petroleum.

Water and energy are intertwined in such a way that if one faces a crisis, then the adequacy and safety of the other is also deeply affected. And both are under stress. We must address the rise in the global demand for energy, while at the same time reduce greenhouse gas emissions to restrain global warming. Demand for water is also increasing rapidly. Clean water reserves are drying up, and the world is facing water crises that are projected to worsen, posing threats to energy production internationally.

Earlier this year, Cape Town, South Africa, made headlines when a three-year drought brought the city close to Day Zero - the day that water stops flowing for its citizens. Israel announced in April 2017 that it will build two new desalination plants, after a five-year drought has caused the worst shortage of natural water the country has seen in a century. Iran is being affected by a decade of low precipitation, with more than $90 \%$ of its population and economic production located in areas of high water stressa situation that bears ominous similarities to what occurred in Syria before the war broke out in 2011. In Flint, Mich., a change in water resource in 2014 resulted in the leaching of lead from old pipes, threatening widespread lead poisoning to its residents.

From a global perspective, such incidents are a warning of a disrupted balance in the nexus between water and energy that we must urgently address. However, this also means that exciting opportunities reside in new materials and materials research to ameliorate these challenges. Two US Department of Energy (DOE) reports, "The Water-Energy Nexus: Challenges and Opportunities" (2014) and "Basic Research Needs for Energy and Water" (2018) focus on this water-energy connection and the wide range of energy and water challenges, and identify "the transformative, fundamental and use-inspired science on which novel solutions

will be founded." Among the top priority research directions in the 2018 report is the challenge of understanding the role of interfaces.

Interfaces, the boundaries between two materials or phases, are unique environments that might be considered a form of sensory nervous system for materials: On and near materials interfaces, reactions and dynamic interactions among water, surfaces, and solutes occur, representing a form of communication between materials. The issue of interfaces crosses many scientific areas and points to potential solutions to water-energy challenges. There is no way to have a selective sensor or a selective sorbent or resilient water treatment membrane or a good catalyst without a thorough understanding of what is occurring at the interface between the water and the surface or molecules with which it interacts. Despite their significance, interfaces in complex aqueous systems remain poorly understood.

Materials science is a gateway to minimizing energy consumption and water consumption, and understanding interfaces is one of the keys that unlocks it. Sensor technology, catalysis, and membrane science are only some of the fields that can contribute to the waterenergy challenge. Close monitoring and probing of the behavior of water and of other species and their interactions at a catalytic or sensor interface is a difficult but necessary task to move things forward.

"Decoding the role of water in processes like catalysis and determining whether it facilitates or inhibits a chemical reaction at an interface is one very promising concept still in its infancy," said Gregory Voth, an expert in theory and simulation of materials, liquids, and biomolecular systems at The University of Chicago. Voth made a distinction between indirect and direct participation of water in reactions. "Water is usually the solvent in which reactions take place, but, it is not a static solvent. It moves and fluctuates, facilitating the movement of the ions and the comeand-go of species from an interface during a reaction," said Voth. In some interesting cases, water itself is a medium. "For example, in the well-known Grotthuss mechanism - used to describe charge (proton) transport-water facilitates proton transfer. An extra proton hops through the water molecules by changing chemical and hydrogen bonds, or water might transiently split and create hydronium $\left(\mathrm{H}_{3} \mathrm{O}^{+}\right)$and hydroxide before it reforms," he said.

Determining the nature of "other species" that are present close to an interface is highly dependent on the water that is being studied. "Because every source of water is unique, and every use of water is unique, and because water is such a fantastic solvent, it can have a million different things in it. Water

Seth B. Darling, Argonne National Laboratory, USA

Eva Karatairi, eva.karatairi@gmail.com 
quality based on the intended end use, that is what we call 'fit for purpose' water, and the ways to assess what is in water or the ways to clean it and reuse it is of great interest," said Voth.

Molecular species in water can be identified using different types of sensors. Their function is based on controlled interactions between molecules at an interface of a sensing material with water. These interactions evoke physicochemical changes (e.g., changes of mass, heat, or electrical potential), which are then converted to an electrical signal, measured, and displayed on a screen. At the University of Wisconsin-Milwaukee, Junhong Chen, professor of mechanical engineering and materials science, is working with his team to develop sensors that can monitor substances in water in real time. "In order to treat the water cost effectively and energy efficiently, we want to know its quality, before and after treatment. This way we can control the treatment process in terms of energy consumption and chemical dosing," he said.

Today, real-time sensors can only detect a couple of factors in drinking water, such as oxygen, and there are no commercial sensors available to monitor key components such as heavy metals, bacteria, or nutrients. Chen's team has targeted lead. "What we want for the sensor we are developing is to be selective toward lead at very low concentrations in drinking water." The US Environmental Protection Agency (EPA) and the Centers for Disease Control and Prevention (CDC) agree that there is no known safe level of lead in a child's blood, which means that the sensor must be highly sensitive to detect quantities of lead well below the action level of $15 \mathrm{ppb}$. The specific sensor that Chen's team is working on consists of a field-effect transistor, with reduced graphene oxide (rGO) sheets acting as the sensing channel. rGO is functionalized with gold nanoparticles, decorated with molecules that act as probes to detect the lead. When lead atoms interact with the probes, they bond through chelation reactions and change the conductivity of the rGO. The change in conductivity (measured as a change in resistance) is proportional to the quantity of metal ion present.

Technologies that reveal what is in the water are a big chapter of water treatment, the other being removal technologies. Choosing a highly efficient catalyst for wastewater treatment, one that can minimize energy consumption and environmental impact, depends on the treatment method itself, said Harold H. Kung, professor of chemical and biological engineering at Northwestern University, who specializes in designing such catalysts. "In general, an iron oxide-based catalyst is a good start no matter whether you choose to use elevated temperature and pressure, with air as the oxidant to oxidize the contaminant, or at near ambient temperature and pressure with hydrogen peroxide as the oxidant. In all cases, the role of a catalyst is to lower the kinetic barrier and speed up the reactions."

The reactions to which Kung refers generate a highly reactive oxidizer that can degrade the contaminant rapidly, including the recalcitrant species (synthetic compounds that are slowly or non-biodegradable). By definition, these highly reactive species have short lifetimes. They react with any organic compounds and begin the degradation process.

Vicki Chen, an expert in membrane separation and head of the School of Chemical Engineering at the University of New South Wales, Australia, has worked the last 10 years on improving membrane performance that can make techniques like pervaporation desalination commercially viable. Pervaporation desalination is a new promising method to remove organics and smaller pollutants (dyes, fumic acids) from water in a more resourcesaving and cost-effective way than reverse osmosis. Vicki Chen explained how things work: "First, we coat the surface of a microporous substrate with a very thin dense polymer, graphene oxide coating, or metal-organic framework materials. We then use low value waste heat to bring the feed stream up to $50^{\circ} \mathrm{C}$ or $60^{\circ} \mathrm{C}$. Water is then selectively adsorbed into a hydrophilic gel or media such as polyvinyl alcohol, diffused through the polymer, and then desorbed on the other side, and removed by vacuum." She added that the water transport through the graphene oxide or metal-organic framework materials is complex, as the microstructured surface can affect the diffusion and evaporation of the water. This technique gives the possibility to treat very difficult solutions, such as mixtures of divalent and monovalent salts with very high salinity (up to a $100 \mathrm{~g} / \mathrm{l}$ salt) (i.e., briny water), which typically cannot be treated by reverse osmosis.

Interpretation of what is going on at interfaces in complex aqueous systems entails far more than one scientific discipline. By merely scratching the surface of current materials science research, one can find that catalysis, sensors, sorbents, and membranes can all contribute significantly to the pressing water and energy nexus problems the global community faces. Although the challenges are great, what is at stake is larger. Fortunately, the outlook in interfacial science research offers hope for a better water-energy future. 